\title{
Regulation of the protein stability of POSH and MLK family
}

\author{
Chunyan Wang, Yang Tao, Yaqing Wang ${ }^{\circledR}$, Zhiheng $X^{\bowtie}$
}

Key Laboratory of Molecular and Developmental Biology, Institute of Genetics and Developmental Biology, Chinese Academy of Sciences, Beijing 100101, China

$\bowtie$ Correspondence: wangyaqing@genetics.ac.cn (Y. Wang), zhxu@genetics.ac.cn (Z. Xu)

Received August 26, 2010 Accepted September 8, 2010

\begin{abstract}
Sequential activation of the JNK pathway components, including Rac1/Cdc42, MLKs (mixed-lineage kinases), MKK4/7 and JNKs, plays a required role in many cell death paradigms. Those components are organized by a scaffold protein, POSH (Plenty of SH3's), to ensure the effective activation of the JNK pathway and cell death upon apoptotic stimuli. We have shown recently that the expression of POSH and MLK family proteins are regulated through protein stability. By generating a variety of mutants, we provide evidence here that the $\mathrm{N}$ terminal half of POSH is accountable for its stability regulation and its over-expression-induced cell death. In addition, POSH's ability to induce apoptosis is correlated with its stability as well as its MLK binding ability. MLK family's stability, like that of POSH, requires activation of JNKs. However, we were surprised to find out that the widely used dominant negative $(d / n)$ form of c-Jun could down-regulate MLK's stability, indicating that peptide from $d / n$ c-Jun can be potentially developed into a therapeutical drug.
\end{abstract}

KEYWORDS the JNK pathway, protein stability, $\mathrm{POSH}$, MLK family and poptosis

\section{INTRODUCTION}

The JNKs (c-Jun N-terminal/stress-activated kinases) have been shown to play major roles in mediating both neuronal and non-neuronal apoptotic cell death (Lei and Davis, 2003; Liu and Lin, 2005). JNK-mediated phosphorylation enhances activation of the inducible transcription factor c-Jun and/or the BH3-only domain protein, BIM (Davis, 2000; Xu et al., 2001), and it has been indicated as a central event in JNK-mediated cell death in response to a plethora of extracellular stimuli (Lei and Davis, 2003).

A cascade pathway has been identified to mediate JNK activation in response to apoptotic stimuli. In this cascade, the GTP-bound forms of Rac1 and/or Cdc42 initiate a sequence of kinase phosphorylation/activation consisted of the mixed lineage kinases (MLKs), MAP kinase kinases (MKKs) 4 and 7 and JNKs (Coso et al., 1995; Xu et al., 2001).

Identified as a binding partner of the activated form of Rac1 (Tapon et al., 1998), POSH evokes death of both nonneuronal (Tapon et al., 1998) and neuronal cells (Xu et al., 2003) when overexpressed. NGF withdrawal and DNA damage in primed $\mathrm{PC} 12$ cells induce the expression of $\mathrm{POSH}$ (Xu et al., 2005). Interfering POSH expression using either anti-sense RNA or siRNA protects neuronal PC12 cells and sympathetic neurons from death induced by NGF withdrawal (Xu et al., 2003) and suppresses the elevation of intracellular phospho-C-Jun levels. It indicates that POSH plays a role in the activation of JNKs, c-Jun phosphorylation and apoptotic cell death. Furthermore, we found that POSH acts as a scaffold protein to organize components of the JNK pathway, including Rac1/Cdc42, MLKs, MKK4 and 7, and JNKs, to ensure the effective activation of the JNK pathway and apoptosis in the presence of apoptotic stimuli (Xu et al., 2003).

We have provided evidence recently for a self-amplifying, feed-forward loop mechanism in which apoptotic stimuli lead to enhanced stability and expression of multiple JNK pathway components including POSH, MLKs, and JIPs (Xu et al., 2005). These effects require JNK activity and are propagated through the pathway itself. However, detailed characterization of $\mathrm{POSH}$ and MLKs is not explored yet. By generating a variety of mutants and using selective inhibitors, we provide evidence here that the $\mathrm{N}$-terminal half of $\mathrm{POSH}$ plays a major role in the regulation of POSH's stability and its ability to 
induce cell death. In addition, POSH's ability to induce apoptosis and its stability are likely to depend on its interaction with MLK family. Furthermore, some interesting findings regarding MLK stability are included.

\section{RESULTS}

\section{$\mathrm{N}$-terminal half of $\mathrm{POSH}$ plays a major role in $\mathrm{POSH}$ induced cell death}

$\mathrm{POSH}$ is essential for cell death in various death paradigms (Xu et al., 2003). To find out which part of POSH is required for its role in cell death, we first cloned the $\mathrm{N}$ - and $\mathrm{C}$-terminal half of POSH separately into the pCMS-EGFP vector (Fig. 1A). Both of those constructs and wild type (wt) $\mathrm{POSH}$ were transfected individually into neuronal differentiated PC12 cells. It is interesting to notice that the $\mathrm{N}$-terminal half of $\mathrm{POSH}$ (POSH 459) induced more apparent cell death than wt $\mathrm{POSH}$ (Fig. 1B). On the contrary, the C-terminal half of $\mathrm{POSH}$ (POSH $\triangle$ N452) did not induce cell death. This indicates that the $\mathrm{N}$-terminal half of $\mathrm{POSH}$ is required for $\mathrm{POSH}$-induced cell death while the C-terminal half of $\mathrm{POSH}$ may play a negative role in $\mathrm{POSH}-\mathrm{evoked}$ cell death. Another possibility is that other proteins, such as AKT family, bind to the Cterminal half of $\mathrm{POSH}$ to inhibit $\mathrm{POSH}$-induced cell death (Cui et al., in preparation).

Since the N-terminal half of $\mathrm{POSH}$ is required for $\mathrm{POSH}$ induced cell death, we decided to focus on this region to gain more insights into the function of $\mathrm{POSH}$. Shorter forms of $\mathrm{N}$ terminal $\mathrm{POSH}$ (deletion of roughly one domain at a time) were cloned into pCMS-EGFP as indicated in Fig. 1A and their ability to evoke death in neuronal differentiated PC12 cells was studied. As shown in Fig. 1B, the shortest POSH fragment, POSH 114, which contains the N-terminal 114 aa with the Ring finger (RF) domain in it, is unable to induce cell death. POSH 254, the N-terminal 254 aa fragment with RF and the first two $\mathrm{SH} 3$ domains, can induce cell death but not as apparent as POSH 459. The effect of POSH 154 on cell death is between that of POSH 114 and POSH 254 (Fig. 1B). Compared with POSH 459, POSH 254 only lacks the Rac1 binding domain. Rac1 and Cdc42 have been shown to be able to induce the activation of MLKs (Böck et al., 2000). Our data indicates that the Rac1 binding domain may play a role in $\mathrm{POSH}$-induced cell death, in accordance with our former model that POSH binds both Rac1 (Cdc42, data not shown) and MLKs to induce activation of the JNK pathway and cell death (Xu et al., 2003).

\section{The N-terminal half of POSH is responsible for $\mathrm{POSH}$ instability}

Apoptotic stimuli increase cellular levels of $\mathrm{POSH}$ through protein stabilization (Xu et al., 2005). As the N-terminal half of $\mathrm{POSH}$ possesses a RF domain to regulate $\mathrm{POSH}$ stability through the proteasomal pathway (Xu et al., 2003) and plays

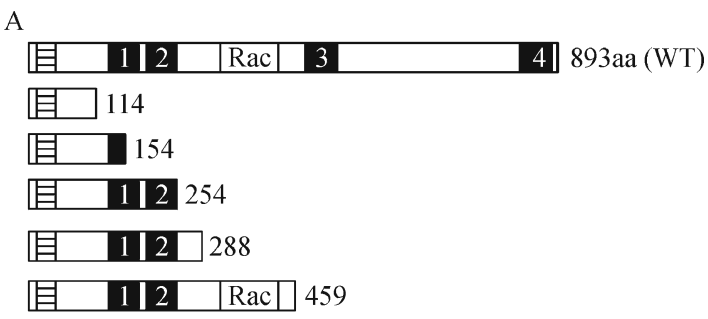

\begin{tabular}{|l|l|}
\hline 3 & $4 \mathrm{~N} 452$ \\
\hline
\end{tabular}

\begin{tabular}{|c|c|c|}
\hline 目 & Rac & $\begin{array}{lllll}1 & 2 & 3 & 4\end{array}$ \\
\hline $\begin{array}{l}\text { ring } \\
\text { finger }\end{array}$ & $\begin{array}{l}\text { Rac-binding } \\
\text { region }\end{array}$ & SH3 domains \\
\hline
\end{tabular}

B

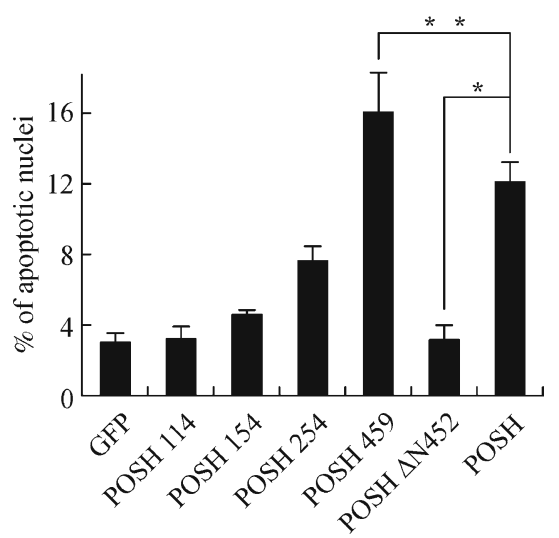

Figure 1. Induction of cell death by POSH mutants. (A) $\mathrm{POSH}$ mutants used in this figure. (B) Induction of apoptosis in neuronal PC12 cells by overexpression of POSH mutants. Neuronally differentiated PC12 cells were transfected with empty vector (pCMS.EGFP, "GFP" in figure) or different POSH constructs in the same vector. Two days after transfection, the percentages of apoptotic nuclei were determined by scoring (per condition) at least 100 nuclei stained with Hoechst 33258 of cells expressing EGFP. Values are the means of three independent experiments $\pm \operatorname{SEM}\left({ }^{*} P<0.01,{ }^{* *} P<0.05\right)$.

a major role in $\mathrm{POSH}$-induced cell death, we set to investigate the regulation of its stability.

We initially found that POSH 459 is more stable than full length $\mathrm{POSH}$, which suggests that the stability of $\mathrm{POSH}$ is correlated with POSH's role in apoptosis (Fig. 2A). To prove this hypothesis, we examined the stability of shorter forms of POSH (Fig. 2). Since MLKs can stabilize POSH (Xu et al., 2005), those POSH constructs were transfected either alone or with MLK2. As shown in Fig. 2A, the shortest form of $\mathrm{POSH}$ (POSH 114) is very unstable. This indicates that the $\mathrm{N}$ terminal 114 aa of $\mathrm{POSH}$ is sufficient for the unstable nature of POSH. Although POSH 114 can be stabilized by MLK2, the stabilization level is much less than the longer forms. $\mathrm{POSH}$ 254 is much more stable than POSH 114 and so is the stabilization induced by MLK2. The stability of POSH 154 is between that of POSH 114 and POSH 254 and its inducible 
stability by MLK2 is also between them. POSH 459 is the most stable form and can be stabilized by MLK2 more obvious than others, even the wt POSH (Fig. 2A, short exposure). The above data suggests that the stability of $\mathrm{POSH} \mathrm{N}$-terminal mutants as well as their stabilization induced by MLK2 is associated with their ability to induce cell death.

\section{POSH stability is correlated with its interaction with MLKs}

Because POSH N-terminal mutant's stability is associated with their stabilization induced by MLK2 (Fig. 2A) and we have shown previously that $\mathrm{POSH}$ and MLKs interact and regulate each other's stability, we reasoned that the stability of POSH might depend on their ability to bind MLKs. This was the case when we did co-immunoprecipitation experiment. Since the longer forms of POSH are more abundant when cotransfected with MLKs, less cell lysates from those cells were used so that roughly equal amounts of $\mathrm{POSH}$ were precipitated down from each sample. As shown in Fig. 2B, the amount of POSH mutant-bound MLK2 is directly associated with $\mathrm{POSH}$ mutants' inducible stabilization by MLK2, although the amount of MLK2 in cell is roughly the
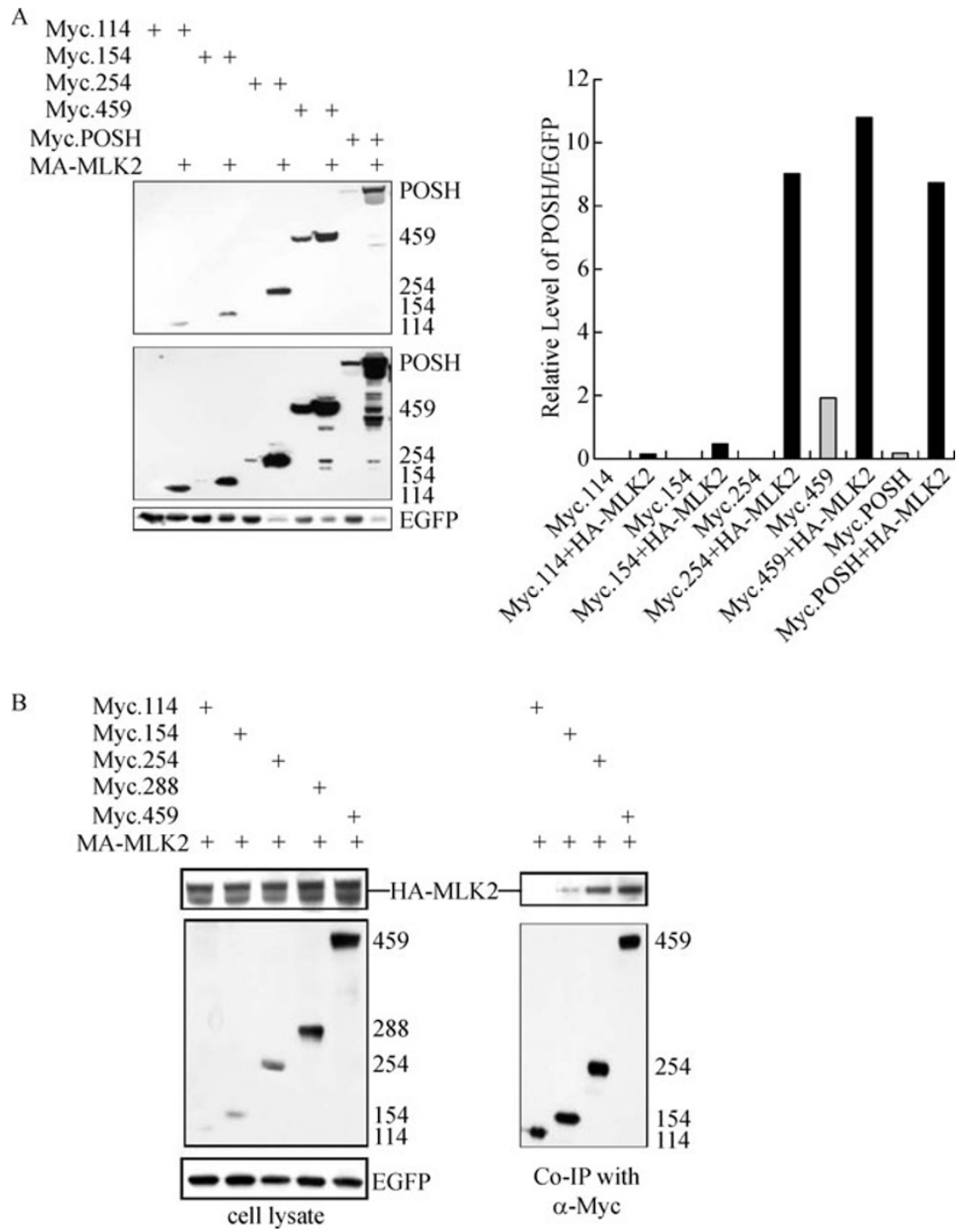

Figure 2. Stability of POSH mutants and their regulation by MLK2. 293 cells were transfected with different Myc-tagged POSH constructs in pCMS.EGFP and HA-tagged MLK2 in pcDNA3, either alone or in combination as indicated. (A) Cell lysates were analyzed $20 \mathrm{~h}$ later for the levels of POSH mutants with Myc antibody by Western blotting and exposed for different time length (top: short exposure; bottom: long exposure). The blot was stripped and re-probed with EGFP antibody as a transfection control. (B) Coimmunprecipitation of MLK2 with POSH mutants. Cells were transfected as in (A) and cell lysate were immunoprecipitated with antiMyc antibody and the immunocomplexes were analyzed for MLK2 with anti-HA antibody as indicated (right panel). The membrane was stripped and reprobed with anti-Myc antiserum to detect the presence of different POSH mutants. 
same. Therefore, POSH mutants' stability is correlated with their MLK binding ability.

We have reported before that MLKs can induce POSH phosphorylation and stabilization and JNK activity is required (Xu et al., 2005). To investigate whether MLKs regulate $\mathrm{POSH}$ stability through phosphorylation, we made different point mutations of potential JNK target residues in POSH. Ser127/ Pro128 in POSH is a potential one and it is conserved from fly to human. To test its role in MLK2-induced POSH stabilization, Ser127 in POSH 154 was mutated to Ala (POSH 154 S127A) and co-transfected with MLK2. As shown in Fig. 3A, POSH 154 S127A can only be stabilized by MLK2 to a level similar to POSH 114 but much less obvious than POSH 154, suggesting that Ser127 plays a role in POSH stabilization. Phosphorylation of Ser127 induced by MLK2 was confirmed by in vivo phosphate labeling (Fig. 3B).

We made Ser to Ala mutation of Ser127 in longer forms of

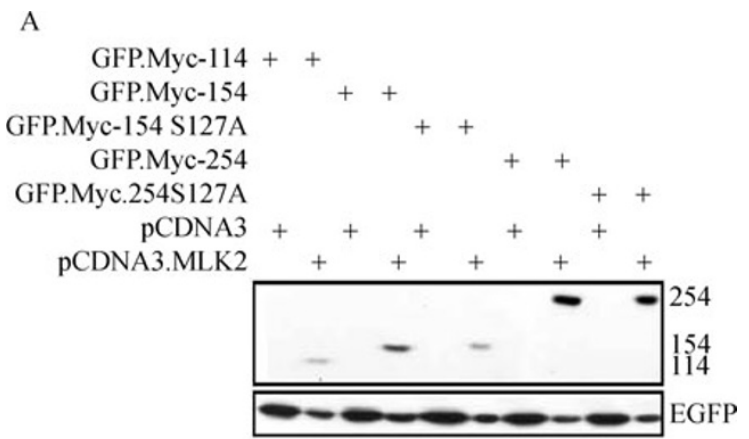

B

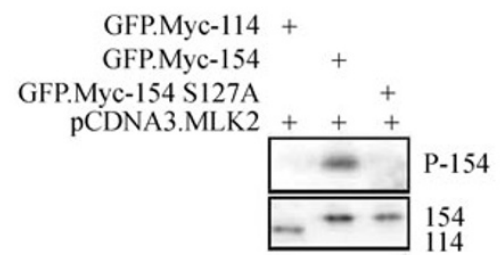

Figure 3. MLK2 induces POSH 154 stabilization through phosphorylation of Ser127. (A) Ser127 is required for MLK2 induced stabilization of POSH 154. Myc tagged POSH 114, POSH 154, POSH 154 S127A, POSH 254 and POSH 254 S127A in $p C M S . E G F P$ (GFP in figure) were separately transfected/co-transfected with pCDNA3.HA-MLK2 into 293 cells respectively. Twenty hours later, cell lysates were analyzed for the levels of POSH mutants by Western blotting with anti-Myc antibody. The blot was stripped and re-probed with eGFP antibody as a transfection control. (B) MLK2 induces the phosphorylation of Ser127. Cells were transfected as in (A). Eighteen hours later, cells were labeled with $\left[{ }^{32} \mathrm{P}\right]$ orthophosphate and cell lysates were subjected to immunoprecipitation. The immuno-complexes were visualized by SDSPAGE followed by autoradiography and subsequent Western blotting with anti-Myc antibody.
$\mathrm{POSH}$ and found that MLK2 has similar effect on the stability of POSH 254 S127A and POSH 254 (Fig. 3A), indicating that Ser127 plays a role in POSH stabilization, but not essential.

\section{SIAH1 and POSH regulate each other's distribution in cells}

We provided evidence recently that expression of SIAH1 evokes cell death through its interaction with $\mathrm{POSH}$ (Xu et al., 2006) and that the subcellular localization of endogenous $\mathrm{POSH}$ changes from diffused pattern to perinuclear dots in the presence of apoptotic stimuli (Kukekov et al., 2006). We therefore tested whether they co-localize with each other in vivo. Full length SIAH1 and SIAH1 S41/S44 were cloned as C-terminal fusion proteins with EGFP while full length $P O S H$ cDNA was cloned as C-terminal fusion proteins with EGFP or $R F P$. The constructs were transfected into MEF cells either separately or together. Cells were also co-transfected with vector containing RFP alone to visualize the entire cell (Fig. 4A and 4C).

As shown in Fig. $4 \mathrm{~A}-\mathrm{C}$, both $\mathrm{SIAH} 1$ and $\mathrm{POSH}$ are diffusely distributed throughout the cell when expressed separately. Previous reports have identified SIAH1 in both nuclei and cytoplasm. Expression of SIAH1 S41/S44 was much stronger than that of wt SIAH1 (Fig. 4B; note the difference in time of exposure) and showed a partially punctuate pattern. Co-expression of POSH and SIAH1 showed that POSH and SIAH1 not only co-localized with each other, but also produced a very distinct pattern with each protein co-localizing to perinuclear dots (Fig. 4D). This indicates that SIAH1 and $\mathrm{POSH}$ regulate each other's distribution in cells and the interaction may contribute to their stabilization (Xu et al., 2006).

\section{Suppression of MLK family stability by JNK inhibitors and $d / n$ c-Jun}

Our previous data indicates that MLKs, similar to $\mathrm{POSH}$, regulate their own stability through a feed forward loop. High concentration $(20 \mu \mathrm{M})$ of SP600125, a selective JNK inhibitor was used to prove that activation of JNKs is required for the stabilization and activation of several MLK family members (Xu et al., 2005). Here, we tested more members of the family at various concentrations of SP600125 and compared them with 200 nM CEP-1347, a selective MLK family inhibitor used to block apoptotic stimuli induced cell death (Xu et al., 2001).

As shown in Fig. 5A and 5B, MLK1 and MLK3 respond to SP600125 in a dose response way, with their protein stability and activity toward phosphorylation of JNK inhibited more than $90 \%$ at $20 \mu \mathrm{M}$. SP600125's effect was detectable even at $1-2.5 \mu \mathrm{M}$. The effect of CEP-1347 at $200 \mathrm{nM}$ is much stronger than that of $20 \mu \mathrm{M}$ SP600125. 2.5 $\mu \mathrm{M}$ SP600125 has some effect on the stability and activity of DLK and MLK2 (Fig. 5A-D and data not shown). However, the effect of $20 \mu \mathrm{M}$ 

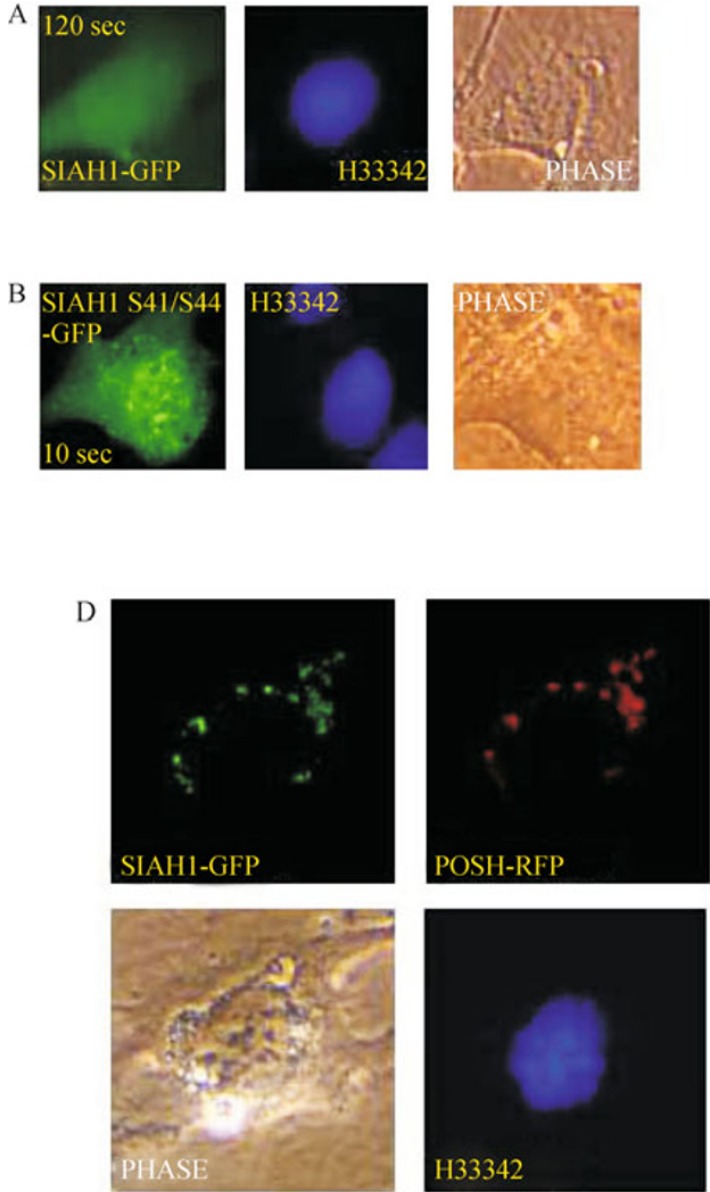
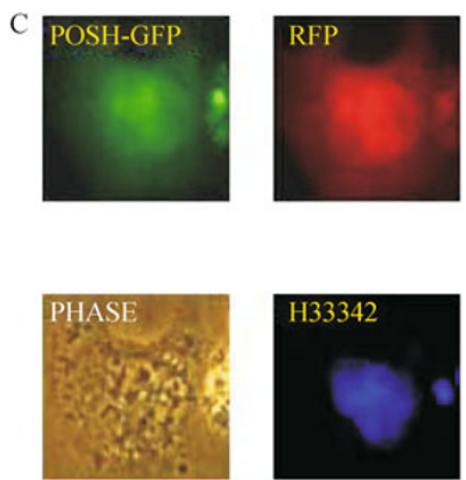

Figure 4. SIAH1 and POSH co-localize in cells. MEF cells were co-transfected/transfected with expression vectors encoding (A) eGFP-SIAH1 fusion protein and red fluorescent protein (RFP); (B) eGFP-SIAH1 S41/S42 fusion protein; (C) eGFP-POSH fusion protein and RFP; (D) eGFP-SIAH1 fusion protein and RFP-POSH fusion protein. $30 \mathrm{~h}$ after transfection, cells were fixed and visualized by microscopy or stained with Hoechst dye 33342 to visualize nuclei. Scale bars, $10 \mu \mathrm{m}$.

SP600125 on DLK and MLK2 is not as evident as it is on MLK1 and MLK3. In addition to suppression of MLKs-induced JNK activation, SP600125 can suppress UV-induced JNK activation in a dose response way (Fig. 5E).

We have shown that $d / n$ c-Jun can suppress MLK family and $\mathrm{POSH}$-evoked cell death, indicating that MLK family and $\mathrm{POSH}$ induce cell death by activating JNKs and subsequently the transcriptional activity of c-Jun (Xu et al., 2001, 2003). This led us to question whether $d / n$ c-Jun can suppress MLKs-induced activation of JNKs by co-expressing MLKs with $\mathrm{d} / \mathrm{n}$ c-Jun. To our surprise, $\mathrm{d} / \mathrm{n}$ c-Jun not only suppressed MLKs-induced phosphorylation of JNKs, it also repressed the expression of MLKs (Fig. 6 and data not shown). Since the MLKs are driven by CMV promoter, it indicates that $d / n$ c-Jun can downregulate MLK protein stability.

We have shown previous that the activity of JNK is required for the stabilization of MLKs and Gallo's group has reported that JNK phosphorylates MLK3 to regulate MLK3's stability. $\mathrm{d} / \mathrm{n}$ c-Jun can interact with JNKs and block the activation of
JNK, therefore, $\mathrm{d} / \mathrm{n}$ c-Jun is likely to destabilize MLK family members indirectly through inhibiting the activity of JNKs.

\section{DISCUSSION}

POSH-induced cell death is correlated with its stability and its interaction with MLKs

The starting point of our investigation was our previous observation that $\mathrm{POSH}$ is required in different cell death paradigms triggered by growth factor deprivation or DNA damage (Xu et al., 2003). We extended the finding and showed here that the $\mathrm{N}$-terminal half of $\mathrm{POSH}$ plays a major role in cell death while the C-terminal half of $\mathrm{POSH}$ may negatively regulate this role. We are currently studying a protein that binds to the $\mathrm{C}$-terminal half of $\mathrm{POSH}$ and try to figure out how it suppresses $\mathrm{POSH}$ expression-induced cell death.

This study generates several interesting points after 
A

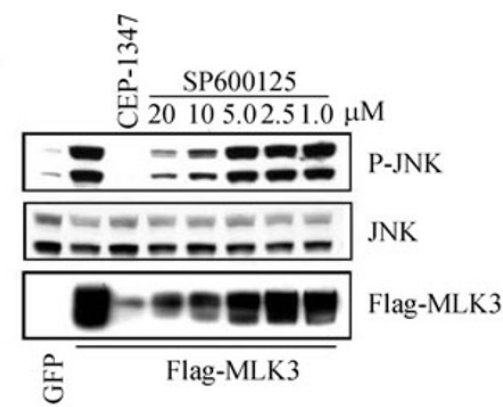

B

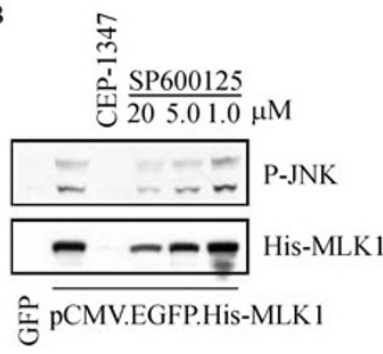

$\mathrm{E}$
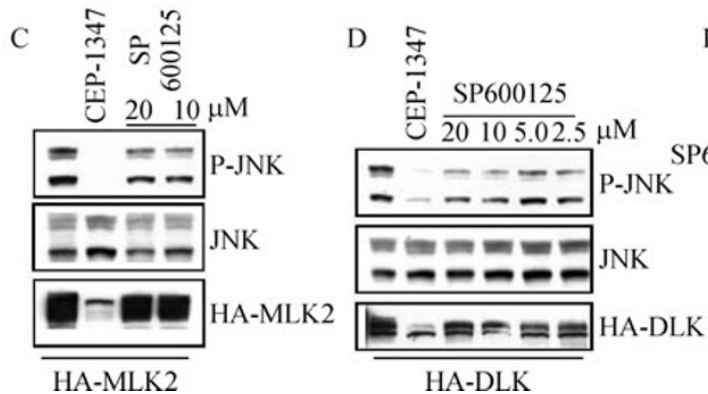

CEP-1347 + SP600125 ( $\mu \mathrm{M}) 01.2520$

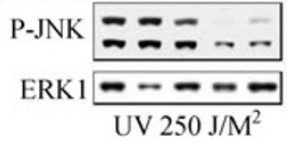

Figure 5. Suppression of MLK family stability/activity and UV induced JNK activation by JNK inhibitor. (A-D) 293 cells were transfected with different MLKs; $4 \mathrm{~h}$ later, CEP-1347 and SP600125 was added to reach the final concentration as indicated. Twenty hours later, cell lysates were analyzed for the levels of MLKs levels by Western blotting with anti-Flag antibody for MLK3, anti-His antibody for MLK1 and anti-HA antibody for MLK2 and DLK. Blots were reprobed with P-JNK, striped and reprobed with JNK as loading control. (E) PC12 cells were pretreated in the presence of different concentration of SP600125 ( $\mu$ M), or CEP-1347 $(200 \mathrm{nM})$ for two hours as indicated. Cells were then treated with UV. Cell lysates were analyzed for P-JNK levels by Western blotting. Blot was striped and reprobed with anti-ERK1 as loading control.

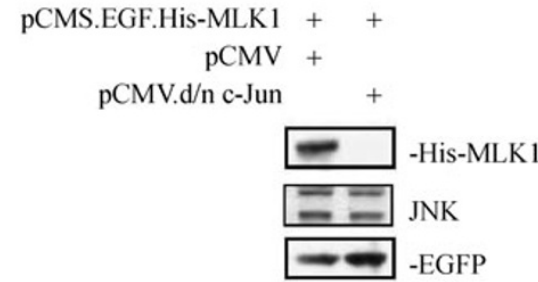

B

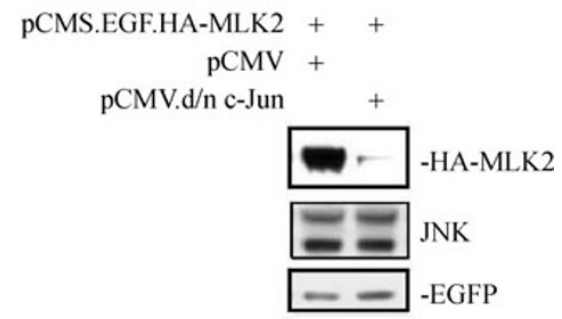

Figure 6. Inhibition of MLK family stability by $\mathrm{d} / \mathrm{n}$ c-Jun. MLK1 (A) or MLK2 (B) in pCMS.EGFP was separately transfected/co-transfected with $p C M V . d / n$ c-Jun into 293 cells. Twenty hours later, cell lysates were analyzed for the levels of MLKs by Western blotting with anti-His antibody for MLK1 (A) and anti-HA antibody for MLK2 (B). The blot reprobed with EGFP antibody as a transfection control and JNK as loading control (A, B). analysis of different mutant forms of POSH. First, The ability for the N-terminal half of $\mathrm{POSH}$ to evoke cell death is correlated with their length from the $\mathrm{N}$ terminus with the following sequence: POSH $114<\mathrm{POSH} 154<\mathrm{POSH} 254<$ $\mathrm{POSH} 459$. This indicates that the first two $\mathrm{SH} 3$ domains and Rac1 binding domain, but not the Ring finger domain, play a role in $\mathrm{POSH}$-induced cell death. Second, the stability of $\mathrm{POSH}$ mutant seems to correlate with their ability to evoke cell death and their potential in interacting with MLKs. In addition, the levels of POSH mutants stabilized by MLKs correlate with POSH mutants' ability to bind MLKs. This further supports our previous hypothesis that $\mathrm{POSH}$ induces cell death through interaction with MLKs and activation of the JNK pathway.

\section{Suppression of MLK family activity and stability by JNK inhibitor and $\mathrm{d} / \mathrm{n} \mathrm{c}$-Jun}

Although there is no report available to distinguish different MLK family members, our data indicates that there is some difference between MLKs in their response to SP600126. It seems that SP600126 has similar effect on MLK1 and MLK3 stability and activity and the effect is different on MLK2 and DLK. This notion is further supported by protein blast search showing that protein homology between MLK3 and MLK1 is higher than that between MLK3 and MLK2 or DLK. 
$\mathrm{d} / \mathrm{n}$ c-Jun has been widely used to effectively suppress different JNK activating apoptotic stimuli induced cell death. The general recognized underlying mechanism is that $d / n c-$ Jun suppresses transcription of apoptotic genes induced by endogenous c-Jun. We provide evidence here that $d / n$ c-Jun suppresses cell death through an alternative but more proficient way, i.e., inhibiting c-Jun activity by blocking the activity and stability of upper stream kinases-MLKs.

A short peptide from JIP1 has been on clinical trial to treat several diseases by competitively blocking the interaction between JNKs and JIP1 and therefore, the activation of the JNK pathway. Our data indicate that peptide from $\mathrm{d} / \mathrm{n}$ c-Jun can be developed into a drug for therapeutic use. It can be potentially more effective as it can block JNK activity from both upstream and downstream.

\section{MATERIALS AND METHODS}

\section{Materials}

CEP-1347 was kindly provided by CEPHALON Inc. Cell growth media RPMI 1640, DMEM, and LipofectAMINE 2000 were purchased from Life technologies, Inc (Frederick, MD). Other primary immunological reagents were directed against JNK, phospho-JNK (Thr183/ Tyr185), MLK3, (New England Biolabs, Beverly, MA), His tag (Novagen, Madison, MI), HA tag (Clontech, Palo Alto, CA), Myc tag and eGFP (Santa Cruz, Santa Cruz, CA). All secondary antibodies were purchased from Pierce (Rockford, IL).

\section{cDNA constructs in mammalian expression vectors}

The following constructs were generously provided as follows: pCDNA3.Flag-SIAH1, pCDNA3.HA-SIAH1 and pCDNA3.FlagSIAH1 S41/S44 were from Eric R. Fearon (University of Michigan Medical Center, Ann Arbor, MI), and dominant negative c-Jun (d/n cJun or Tam67 c-Jun) was from Michael J. Birrer (NIH, Rockville, MD).

MLK1, MLK2, MLK3, DLK and their kinase inactive forms in pCDNA3 and pCMS-EGFP have been described previously (Xu et al., 2001), as has wt POSH construct in pCMS-EGFP (Xu et al., 2003). Myc tagged POSH 114 (coding aa 1-114), POSH 154 (coding aa 1-154), POSH 254 (coding aa 1-254), POSH 459 (coding aa 1-459), and POSH $\Delta \mathrm{N} 452$ (coding aa 452-end) were constructed by PCR amplification of the respect coding sequence from pCMS-EGFP. Myc-POSH with proper primers that has additional stop code for POSH 114, POSH 154, POSH 254, and POSH 459. pCMS-EGFP. Myc-POSH 154 127A and pCMS-EGFP. Myc-POSH 254 127A were made by site-directed mutagenesis (Stratagene).

pEGFP.SIAH1 and pEGFP.SIAH1 S41/S44 were constructed by PCR amplification of the corresponding cDNAs in $p C D N A 3$ with primers 5'-AAGCTTAACCATGAGCCGCCAGACTGCTACAGCA-3' and 5'-GAATTCGACACATGGAAATAGTTACATTGAT-3', cloned into pCR2.1.TOPO and then sub-cloned into the EcoRI/Hindlll site of pEGFP-N1. pEGFP.POSH was constructed by PCR amplification of $P O S H$ from $p C M S-E G F P . M y c-P O S H$ (Xu et al., 2003) with primers $5^{\prime}$ CCGCTCGAGGCCACCATGGATGAGTCTGCCTTGTTGGAC-3' and 5'- GATTGGATCCATGTTTTCCACAAAGCTCCCTGG-3', cloned into $p C R 2$.1.TOPO and then sub-cloned into the $\mathrm{Xhol} / \mathrm{BamHI}$ site of $p E G F P-N 1$. The POSH fragment was excised from $p E G F P-P O S H$ with $\mathrm{Nhel} / \mathrm{BamHI}$ and cloned into the same sites of $p D S R e d 1-N 1$ to construct $p$ Red-POSH.

Cell culture, transfections, metabolic labeling, co-immuno precipitation and Western blotting

PC12 and 293 cell culture and transfection were described previously (Xu et al., 2001, 2003). Transfected 293 cells were metabolically labeled with ${ }^{32} \mathrm{P}$ orthophosphate for $3 \mathrm{~h}$ and extracted with Triton X-I00 lysis buffer as described elsewhere (Aletta et al., 1989). Coimmunoprecipitation and Western immunoblotting were performed as previously described (Xu et al., 2001, 2003).

\section{ACKNOWLEDGEMENTS}

We thank Drs. R. Davis, and A. Hall for providing valuable reagents. This work was supported in part by grants from Ministry of Science and Technology of China, the National Basic Research Program of China (973 Program) (Grant Nos. 2007CB947202 and 2006CB500701), and the National Programs for High Technology Research and Development Program of China (863 Program) (Grant Nos. 2006AA02Z173 and 2009DFA32450); National Natural Science Foundation of China (Grant Nos. 30725007, 30870527 and 30670663); Chinese Academy of Sciences (Bairen plan and Grant No. KSCX1-YW-R-62/84).

\section{REFERENCES}

Aletta, J.M., Shelanski, M.L., and Greene, L.A. (1989). Phosphorylation of the peripherin $58-\mathrm{kDa}$ neuronal intermediate filament protein. Regulation by nerve growth factor and other agents. J Biol Chem 264, 4619-4627.

Böck, B.C., Vacratsis, P.O., Qamirani, E., and Gallo, K.A. (2000). Cdc42-induced activation of the mixed-lineage kinase SPRK in vivo. Requirement of the $\mathrm{Cdc} 42 / \mathrm{Rac}$ interactive binding motif and changes in phosphorylation. J Biol Chem 275, 14231-14241.

Coso, O.A., Chiariello, M., Yu, J.C., Teramoto, H., Crespo, P., Xu, N., Miki, T., and Gutkind, J.S. (1995). The small GTP-binding proteins Rac1 and Cdc42 regulate the activity of the JNK/SAPK signaling pathway. Cell 81, 1137-1146.

Davis, R.J. (2000). Signal transduction by the JNK group of MAP kinases. Cell 103, 239-252.

Figueroa, C., Tarras, S., Taylor, J., and Vojtek, A.B. (2003). Akt2 negatively regulates assembly of the POSH-MLK-JNK signaling complex. J Biol Chem 278, 47922-47927.

Kukekov, N.V., Xu, Z., and Greene,L.A. (2006). Direct interaction of the molecular scaffolds $\mathrm{POSH}$ and JIP is required for apoptotic activation of JNKs. J Biol Chem 281, 15517-15524.

Lei, K., and Davis, R.J. (2003). JNK phosphorylation of Bim-related members of the Bcl2 family induces Bax-dependent apoptosis. Proc Natl Acad Sci U S A 100, 2432-2437.

Liu, J., and Lin, A. (2005). Role of JNK activation in apoptosis: a double-edged sword. Cell Res 15, 36-42.

Tapon, N., Nagata, K., Lamarche, N., and Hall, A. (1998). A new rac target $\mathrm{POSH}$ is an $\mathrm{SH} 3$-containing scaffold protein involved in the JNK and NF-kappaB signalling pathways. EMBO J 17, 1395-1404. 
Xu, Z., Maroney, A.C., Dobrzanski, P., Kukekov, N.V., and Greene, L. A. (2001). The MLK family mediates c-Jun N-terminal kinase activation in neuronal apoptosis. Mol Cell Biol 21, 4713-4724.

Xu, Z., Kukekov, N.V., and Greene, L.A. (2003). POSH acts as a scaffold for a multiprotein complex that mediates JNK activation in apoptosis. EMBO J 22, 252-261.
Xu, Z., Kukekov, N., and Greene, L. (2005). A stability-based selfamplifying feed forward loop mechanism for regulation of the apoptotic JNK pathway. Mol Cell Biol 25, 9949-9959.

Xu, Z., Sproul, A., Wang, W.Y., Kukekov, N., and Greene, L.A. (2006). Siah1 interacts with the scaffold protein POSH to promote JNK activation and apoptosis. J Biol Chem 281, 303-312. 